\title{
Prevalencia de resiliencia y autoestima sobre el rendimiento escolar en estudiantes de instituciones educativas de Ate Vitarte, Lima
}

\author{
Resilience and self-esteem prevalence on educational institutions \\ students' school performance from Ate Vitarte, Lima
}

Edson Jorge Huaire Inacio'

Universidad Nacional de Educación Enrique Guzmán y Valle

\section{RESUMEN}

Objetivos: Determinar la prevalencia y relación entre resiliencia, autoestima y el rendimiento escolar en estudiantes de 3ro, 4to y 5 to grado de educación secundaria de instituciones educativas del distrito de Ate Vitarte en el departamento de Lima. Métodos: Es un estudio descriptivo - correlacional. Se seleccionó una muestra representativa de 233 estudiantes de tres grados de estudio, a quienes se les administró la Escala de Resiliencia (ERA) que consta de 12 ítems divididos en tres áreas, 04 ítems para cada uno, que miden, realización personal, autodeterminación y autoconfianza. Para medir la variable autoestima, se utilizó la Escala de Autoestima de Rosenberg (1965), adaptada a las edades de la muestra por Villalba (1992). Resultados: Existe una diferencia estadística en las frecuencias de estudiantes ubicados en los diversos niveles de resiliencia y autoestima. La revisión señala que el nivel prevalente de resiliencia es alto, allí se ubica el $71,70 \%$ de éstos o 7 de cada 10 . El nivel prevalente de autoestima también es alto, allí se clasifica el $76 \%$ de los estudiantes o 7 de cada 10. El nivel prevalente de rendimiento escolar que caracteriza a la muestra es el nivel bajo; en ello se encuentra el 54,50\% de los estudiantes, o 5 de cada 10. Conclusiones: El nivel de prevalencia tanto de resiliencia como de autoestima de los estudiantes es alto y el de rendimiento escolar es bajo, además, existe una relación estadísticamente significativa entre los niveles de resiliencia, autoestima y rendimiento escolar.

Palabras Clave: Resiliencia, autoestima, rendimiento escolar, realización personal, autodeterminación, autoconfianza.

1 Magíster en Docencia Universitaria por la Universidad Nacional de Educación Enrique Guzmán y Valle, estudios de maestría en Psicología Cognitiva en la Universidad de Buenos Aires, diplomado en Gestión Educativa en la Facultad Latinoamericana de Ciencias Sociales (FLACSO-Argentina). Asesor de la asociación educativa CLARIDAD (España). 


\section{ABSTRACT}

Objectives: To determine the prevalence and relationship among resilience, selfesteem and school performance in third, fourth and fifth grade secondary students from Ate Vitarte district's educational institutions, Lima department. Methods: Correlational descriptive research. A representative sample of 233 students from three grades was selected; the Resilience Scale (RE) was applied, which is formed by 12 items divided into three areas, 04 items for each one, what they measure, personal fulfillment, self-determination and self-confidence. To measure the self-esteem variable, the Rosenberg Self-Esteem Scale (1965) was used, adapted to the sample ages by Villalba (1992). Results: There is a statistically difference in the students frequencies from the different resilience and self-esteem levels. The review shows that the prevailing level is high, there is the $71,70 \%$ of students or 7 out of 10 . The prevailing level of self-esteem is high too, there is the $76 \%$ of students or 7 out of 10 . The prevailing level in academic performance that characterizes the sample is the low level; there is the $54,50 \%$ of students, or 5 out of 10. Conclusions: The prevalence level of both resilience and self-esteem in students is high and in school performance is low, also here exists a significant statistically relationship between resilience levels, selfesteem and school performance.

Keywords: Resilience, self-esteem, school performance, self-realization, selfdetermination, self-confidence.

\section{INTRODUCCIÓN}

En las últimas décadas del siglo $X X$ y lo que va del siglo $X X \mid$ se ha impulsado un nuevo enfoque para entender el desarrollo de los seres humanos, este enfoque está basado en la resiliencia que se caracteriza por la búsqueda de las fortalezas de las personas frente a la adversidad. Durante mucho tiempo, ha prevalecido una tendencia a juzgar a las personas por sus falencias, sus debilidades y por todo lo negativo que se les ocurría. Afortunadamente en los últimos años esta visión fatalista ha sido reemplazada por una consideración más respetuosa y una visión más optimista de las personas (1) y se ha considerado de que las personas tienen la capacidad de construir competencias internas para sobreponerse a las experiencias negativas y fortalecerse en el proceso (2).

Si bien es cierto, que el comportamiento resiliente de las personas está sujeto tanto a factores externos como internos (3), su manifestación es distinta e incluso opuestas para cada sujeto frente a un estímulo - hacia situaciones que le causa algún daño. En consecuencia, lo que tratamos de afirmar es que, existen tipos de reacciones frente a estímulos dolorosos o adversos. Existen personas que frente al dolor o adversidad reaccionan con conductas de vulnerabilidad y debilidad. También, existen personas que permanecen indiferentes frente a un estímulo. Y también, existen personas con respuestas resilientes que frente a un estímulo adverso, resisten, se fortalecen y logran alcanzar adecuados niveles de calidad de vida (4).

En el presente estudio trataremos de desvelar algunas características resilientes de los estudiantes de colegios de nivel socio económico medio-bajo del distrito de AteVitarte, para lo cual el objetivo del estudio fue determinar la prevalencia y relación existente entre el nivel de resiliencia, autoestima y rendimiento escolar.

Desde siempre se sabe, que la escuela ocupa un lugar preponderante y privilegiado en el desarrollo personal como social de los niños y adolescentes, debido a que durante la educación básica, los niños y adolescentes se centran en la escuela y en las amistades, en ella encuentran a sus amigos íntimos o amigas íntimas. Sin embargo, es también el lugar donde están más propensos al riesgo, porque pueden encontrar la amistad o el amigo equivocado. Por algo, Beck (5) señala que las sociedades contemporáneas se han constituido en sociedades de riesgo. Para minimizar estos riesgos, las escuelas se deben convertir en escuelas resilientes, 
donde las interacciones entre estudiantes y profesores o personal implicado en la educación, analizan y previenen los riesgos que se pueden generar en estos contextos, desde estos lugares se deben promover los factores protectores como contraparte a los factores de riesgo considerados en el modelo tradicional como negativos o patológicos, estos factores protectores se deben desarrollar hasta llegar a los factores resilientes, definidos muy claramente por Munist y Suarez (6) como escudos que favorecen el desarrollo normal de los jóvenes y estudiantes.

\section{Resiliencia y autoestima}

La resiliencia es un conglomerado de factores innatos y aprendidos, dentro de este concepto, la autoestima es considerada como un factor esencial y está en estrecha relación con otros elementos como el sentirse valorado, comprendido, estar rodeados de personas que ratifican su valía y les animan a seguir adelante, etc. Si bien es cierto, que son elementos subjetivos que están vinculados a la sensación de bienestar experimentado por cada sujeto, estas características están también relacionados a un bienestar general del estudiante, a afectos positivos, referidos a sentimientos que reflejan una percepción alegre $y$ placentera de la vida (7). En consecuencia, tal como señalan Melillo, Estamatti y Cuestas (8) entre estas dos variables existe una conformación de relación positiva tanto en niños y adolescentes a partir de la relación con el otro; el otro puede ser la madre, el padre, los hermanos, en general es la familia más cercana, le siguen, los amigos, los docentes, y otros cuidadores que le generan un ambiente saludable y de bienestar general.

Desde los estudios de la neurociencia y del desarrollo humano, se sabe que cuando falta una conformación adecuada de soporte familiar, muchos adolescentes buscaran un acercamiento a otros grupos marginales que les otorgan algún tipo de identidad, pertenencía y reconocimiento dentro de su grupo y si estos son aceptados, se sentirán más satisfechos estando con el grupo y no con la familia, solo por el hecho de encontrar algún tipo de confianza y valía para terminar siendo parte de ellos.

Desde esta perspectiva, la escuela tiene la capacidad de influir en los jóvenes, sus familias y el entorno inmediato puesto que es la segunda fuente de seguridad después del hogar y a veces -como mencionaManciaux (9) es la única en los niños. La mayoría de los niños y adolescentes encuentran en su interacción en este medio, las condiciones de protección, seguridad y los modelos que necesitan para desarrollarse (10). Por lo tanto, desde la perspectiva resiliente, la escuela es un lugar adecuado para promover la resiliencia y elevar su autoestima, brindar expectativas de superación permanente a los estudiantes e informales lo necesario para tener éxito (2).

\section{Resiliencia y rendimiento escolar}

En el contexto educativo se concibe a la resiliencia como una aptitud implicada en una serie de actitudes, tales como el esfuerzo gradual y sostenido para lograr objetivos académicos, potenciar la tolerancia a la frustración y la ambigüedad y sobre todo tener la capacidad asertiva para la toma de decisiones (11). Además, la resiliencia permite desarrollar todo un conjunto de competencias genéricas para avanzar en el conocimiento y la construcción de relaciones interpersonales estables, sin temor al error y considerar como un estadio más en la búsqueda de una nueva respuesta positiva.

Evidencias como la de Gallesi y Matalinares (12), manifiestan que los factores personales de resiliencia tiene una relación significativa con el rendimiento académico. Además, estas estarían en relación con el clima institucional del centro educativo, de las interacciones llenas de significado entre el joven en formación y una persona significativa que puede ser el docente o bastaría con el apoyo de un compañero para promover resiliencia (13). De esta manera, es importante hacer notar, sobre todo al personal directivo o a las personas 
encargadas de las políticas educativas, que la construcción de resiliencia en los estudiantes no es "una cosa más", sino que es la base de escuelas eficaces y de lo que constituye una educación de excelencia. La educación excelente dará lugar a la construcción de resiliencia (14).

\section{MATERIAL Y MÉTODOS}

Los participantes están conformados por 233 estudiantes hombres y mujeres de 3ro, 4 to y 5to grado de educación secundaria, las edades oscilan entre los 13 y 18 años de edad, esta muestra pertenece a dos colegios de Ate-Vitarte, Lima.

Respecto a los instrumentos. Para medir el nivel de resiliencia, se utilizó la Escala de Resiliencia (ERA) desarrollado por Omar, (7) que consta de 12 reactivos divididos en tres componentes, cuatro ítems por componentes que miden: realización personal, autodeterminación y autoconfianza; la escala presenta un formato tipo Likert de 5 puntos, variando desde 1 (total desacuerdo) a 5 (totalmente acuerdo).

Para medir autoestima, se utilizó la Escala de autoestima desarrollada por Rosenberg, incluye diez ítems cuyos contenidos se centran en los sentimientos de respeto y aceptación de sí mismo. La mitad de los ítems están enunciados positivamente y la otra mitad en forma negativa. La puntuación de esta escala también es tipo Likert, donde los ítems se responden en valor de cuatro puntos $(1=$ muy de acuerdo, $2=$ de acuerdo, $3=$ en desacuerdo, $4=$ totalmente en desacuerdo). Para su corrección deben invertirse las puntuaciones de los ítems enunciados negativamente $(3,5,8,9,10)$ y posteriormente sumarse todos los ítems. La puntuación total, por tanto, oscila entre 10 y 40 .

Para evaluar el rendimiento escolar de los estudiantes, se ha recurrido a las calificaciones promedios de Matemática y Comunicación ya que es considerado según la UNESCO, como el indicador más confiable para medir el nivel de aprovechamiento escolar de los estudiantes. Esta clasificación se realizó siguiendo la escala vigesimal propuestos por Reyes: alto (15-20), medio (13-14,99), bajo (11$12,99)$ y deficiente: $(0-10,99)$.

La aplicación de los instrumentos se llevaron a cabo de manera colectiva en los salones de clase de los estudiantes, la duración fue de aproximadamente 25 minutos para las dos escalas tanto de resiliencia como de autoestima. Antes de iniciar el desarrollo de las escalas, se les dieron las instrucciones y se resolvieron algunas dudas que se presentaron.

\section{RESULTADOS}

\section{Niveles de resiliencia autoestima y rendimiento escolar}

Los resultados de la prueba ji cuadrado de homogeneidad mostrados en la tabla $N^{\circ}$ 1 indican que la referida prueba tiene un resultado estadísticamente muy significativo $(p<0,0001)$; en consecuencia existe una diferencia estadística en las frecuencias de estudiantes ubicados en los diversos niveles de resiliencia. La revisión señala que el nivel

Tabla $N^{\circ}$ 1: Resultados de la aplicación de la prueba ii cuadrado de homogeneidad.

\begin{tabular}{cccc}
\hline $\begin{array}{c}\text { Valor } \mathrm{ii} \\
\text { cuadrado de } \\
\text { homogeneidad }\end{array}$ & $\begin{array}{c}\text { Grado } \\
\text { de }\end{array}$ & $\begin{array}{c}\text { Nivel } \\
\text { le }\end{array}$ & Significatividad \\
& significación () & \\
\hline 429,468 & 4 & 0,0001 & $\mathrm{Si}$ \\
\hline
\end{tabular}

Tabla $N^{\circ}$ 2: Nivel de resiliencia.

\begin{tabular}{lcc}
\hline Niveles & Frecuencia & Porcentaje \\
\hline Muy Bajo & 1 & 0,40 \\
Bajo & 4 & 1,70 \\
Medio & 54 & 23,20 \\
Alto & 167 & 71,70 \\
Muy Alto & 7 & 3,00 \\
TOTAL & 233 & $100 \%$ \\
\hline
\end{tabular}


Tabla $N^{\circ}$ 3: Nivel de autoestima

\begin{tabular}{lcc}
\hline \multicolumn{1}{c}{ Niveles } & Frecuencia & Porcentaje \\
\hline Muy Bajo & 0 & 0 \\
Bajo & 0 & 0 \\
Medio & 31 & 13,30 \\
Alto & 177 & 76,00 \\
Muy Alto & 25 & 10,70 \\
TOTAL & 233 & $100 \%$ \\
\hline
\end{tabular}

Tabla N 4: Resultados de la aplicación de la prueba ji cuadrado de homogeneidad.

\begin{tabular}{cccc}
\hline $\begin{array}{c}\text { Valor ii } \\
\text { cuadrado de } \\
\text { homogeneidad }\end{array}$ & $\begin{array}{c}\text { Grado } \\
\text { de }\end{array}$ & $\begin{array}{c}\text { Nivel } \\
\text { de }\end{array}$ & Significatividad \\
significación () & & \\
\hline 190,798 & 2 & 0,0001 & $\mathrm{Si}$ \\
\hline
\end{tabular}

Tabla N ${ }^{\circ}$ 5: Nivel de rendimiento escolar.

\begin{tabular}{lcc}
\hline Niveles & Frecuencia & Porcentaje \\
\hline Deficiente $(0-10,99)$ & 31 & 13,30 \\
Bajo $(11-12,99)$ & 127 & 54,50 \\
Medio $(13-14,99)$ & 46 & 19,70 \\
Alto $(15-20)$ & 29 & 12,50 \\
TOTAL & 233 & $100 \%$ \\
\hline
\end{tabular}

Tabla N ${ }^{\circ}$ 6: Resultados de la aplicación de la prueba ii cuadrado de homogeneidad.

\begin{tabular}{cccc}
\hline $\begin{array}{c}\text { Valor Ji } \\
\text { Cuadrado de } \\
\text { Homogeneidad }\end{array}$ & $\begin{array}{c}\text { Grado } \\
\text { de } \\
\text { libertad }\end{array}$ & $\begin{array}{c}\text { Nivel } \\
\text { de } \\
\text { significación () }\end{array}$ & Significatividad \\
\hline 111,155 & 3 & 0,0001 & $\begin{array}{c}\text { Si, altamente } \\
\text { significativo }\end{array}$ \\
\hline
\end{tabular}

prevalente es alto, allí se clasifica el 71,70 $\%$ de éstos $\circ 7$ de cada 10 , tabla $N^{\circ} 2$.

\section{Identificación del nivel de rendimiento escolar}

Para identificar el nivel de rendimiento escolar que caracteriza a los estudiantes del 3ro, 4to y 5to grado de educación secundaria del distrito de Ate Vitarte, se trató a esta variable como si fuera categórica, clasificándose de acuerdo con la puntuación promedio de la escala vigesimal $(0-20)$. Esta clasificación se realizó atendiendo a los niveles propuestos por Reyes: alto (15-20), medio (13-14,99), bajo (11-12,99) y deficiente: $(0-10,99)$. Posteriormente, se efectuó un análisis de frecuencia porcentual. Con el objeto de tener clara las diferencias observadas en este análisis y precisar el nivel prevalente. En tal efecto, se realizó la prueba ji cuadrado de homogeneidad, bajo el supuesto que de no existir diferencias en este nivel de los sujetos de la muestra, se distribuirían por igual en los diferentes niveles. Sin embargo, el nivel donde se observó la mayor cantidad de sujetos definió el nivel prevalente de rendimiento escolar de los estudiantes.

Los resultados mostrados en la tabla $N^{\circ} 5$ indican que hay una diferencia estadísticamente muy significativa $(p<0,0001)$, entre las frecuencias de estudiantes clasificados en cada uno de los niveles de rendimiento escolar; la revisión permite precisar que el nivel prevalente de rendimiento escolar que caracteriza a la muestra estudiada es el nivel bajo. En ello se encuentra el $54,50 \%$ de los estudiantes, - 5 de cada 10 estudiantes.

\section{Relación de resiliencia y autoestima con rendimiento escolar}

Para determinar la relación que hay entre estas tres variables se utilizó la prueba estadística paramétrica coeficiente de correlación de Pearson. Previamente se comprobó que los puntajes de las variables siguieran un patrón de distribución normal que es la principal condición para la correcta aplicación de esta prueba estadística. Esta comprobación se hizo empleando la prueba de normalidad de Kolmogorov-Smirnov. En el caso que las distribuciones no fueron normales se podrían aplicar la prueba de coeficiente de correlación no paramétrico Rho de Spearman.

a. Predictores (constante) Resiliencia

b. Predictores (constante) Resiliencia + Autoestima

Utilizando el método stepwise (paso a 
Tabla N 7 : Coeficiente de correlación múltiple (R).

\begin{tabular}{ccccc}
\hline Modelo & $\begin{array}{c}\text { Coeficiente de } \\
\text { correlación } \\
\text { múltiple (R) }\end{array}$ & $\begin{array}{c}\text { Coeficiente } \\
\text { de determinación } \\
\text { múltiple (R2) }\end{array}$ & $\begin{array}{c}\text { Prueba F } \\
\text { (Anova) }\end{array}$ & $\begin{array}{c}\text { Nivel de } \\
\text { significatividad } \\
\text { estadística de } F\end{array}$ \\
\hline 1 & $0,796 \mathrm{a}$ & 0,634 & 399,462 & 0,0001 \\
2 & $0,804 \mathrm{~b}$ & 0,646 & 210,336 & 0,0001 \\
\hline
\end{tabular}

paso) se hallan los resultados mostrados en esta tabla. En primer lugar, el modelo 2 muestra la relación de la resiliencia y la autoestima actuando en conjunto sobre el rendimiento escolar. Este modelo es, según el resultado de la prueba $f$ (análisis de varianza), estadísticamente muy significativo indicando que el coeficiente de correlación múltiple cuantifica una relación estadística muy significativa $(p<0,01)$ entre la ecuación de regresión de ambas variables con el rendimiento escolar.

En segundo lugar, el modelo 1 también tiene un valor $f$ estadísticamente muy significativo $(p<0,01)$, lo que indica que el coeficiente de correlación múltiple cuantifica una relación de este nivel entre resiliencia y rendimiento escolar.

El análisis comparativo de ambos modelos permite observar que el incremento del valor del coeficiente de correlación múltiple que hay del modelo 1 al modelo 2 es bastante bajo (apenas 0,08). Este hecho está en directa relación con el alto coeficiente de correlación de resiliencia con autoestima $(0,629)$ lo que causa una supresión de varianza del rendimiento escolar explicada por la autoestima. En este sentido, para efectos de predicción del rendimiento escolar en la muestra estudiada, bastaría tomar en cuenta a la variable resiliencia. En este último caso, el valor del coeficiente de determinación múltiple (R2) señala el porcentaje de la varianza del rendimiento escolar predicho por esta variable $(63,4 \%)$.

\section{DISCUSIÓN}

Los resultados generales obtenidos en este estudio apuntan a que las variables principales estudiadas resiliencia y autoestima están en relación significativa con la puntuación obtenida en el rendimiento escolar. En tal sentido, se determina que, existe una relación estadísticamente significativa y positiva entre resiliencia, autoestima y rendimiento escolar. Dicha relación se confirma en los resultados globalesy entodos los resultados específicos, cuando se contrastaron los resultados que se obtuvieron de las dimensiones de resiliencia, (realización personal, autodeterminación, autoconfianza) con los resultados generales de autoestima y rendimiento escolar.

Este hallazgo nos permite, en principio, confirmar la interacción significativa y positiva entre las dos primeras variables (resiliencia y autoestima). Esto puede construir un mecanismo explicativo al planteamiento conceptual de la autoestima como uno de los pilares de resiliencia. Al mismo tiempo, considerados por algunos teóricos como un componente de valoración personal del estudiante, en la medida en que el individuo le atribuye valor a sus propias perspectivas, y a sentirse a gusto consigo mismo. Esto también está en función del amor, respeto y aceptación de los otros que ratifican su valía y les animan a seguir; además del propio sentido del valor, competencia y capacidad que uno experimenta de sí mismo (15).

En este sentido, se confirma que la autoestima es un indicador importante de resiliencia en los estudiantes, dado que les hacen sentir más confiados en sus capacidades, les permite desarrollar y potenciar estrategias para enfrentar situaciones de tensión y lograr sin mayores dificultades sus metas. Como "efectos secundarios" positivos, mejora el sentimiento de bienestar y control sobre su propia vida, disminuye su ansiedad, el estrés, aumenta la solidaridad con los demás y sobre todo, mejora su logro académico (16). 
En segundo lugar, estos mismos resultados confirman la relación significativa entre el nivel de resiliencia y rendimiento escolar, es decir, que los sujetos con mayor nivel de resiliencia, experimentan mayor nivel de rendimiento escolar. Dichos hallazgos son congruentes con los reportes de Gallesi y Matalinares (12), Peralta, Ramírez y Castrano (17), Villalta (18), que encuentran que los estudiantes con un puntaje elevado en resiliencia también obtienen un puntaje elevado de rendimiento escolar y que los factores de resiliencia están asociados a los niveles de logro académico. En este sentido se cree que la resiliencia de las personas parece determinar el aumento de la capacidad para resolver problemas de su dominio de conocimiento y favorecer los procesos de cambios positivos en el rendimiento escolar.

Esta mejora en el rendimiento escolar, está vinculado al proceso de mejora en relación tanto a la institución como a los docentes, es decir al clima escolar e institucional. Por una parte, las instituciones que reconocen el esfuerzo de sus docentes, promueven actitudes positivas de los docentes hacia los estudiantes. Por otro lado, los profesores que estimulan $y$ establecen relaciones significativas con sus estudiantes, se acercan a sus estudiantes con una clara vía de acción y con la voluntad de ayudarlos (13).

En cuanto al análisis de los resultados obtenidos entre las variables, autoestima y rendimiento escolar, los datos encontrados son altamente significativas lo que confirma el hallazgo de Arancibia (19), Llinares, Molpeceres y Musitu, (20), Tacconelli (21) entre otros, quienes sustenta que el nivel de autoestima en los escolares es uno de los factores que influyen de manera considerable en el rendimiento escolar, convirtiéndose en un significativo indicador de logro o fracaso del estudiante. También se sustenta que los adolescentes con alta autoestima académica tienden a priorizar en mayor medida valores prosociales, de conformidad y de autodirección, etc.

Los resultados mostrados permiten concluir que el nivel de prevalencia tanto de resiliencia como de autoestima de los estudiantes es alto y el de rendimiento escolar es bajo. Por otro lado, los resultados permiten aceptar nuestra hipótesis principal, en la cual postulamos que existe una relación estadísticamente significativa entre el nivel de resiliencia, autoestima y rendimiento escolar en estudiantes de 3ro, 4to y 5to grado de educación secundaria de Ate Vitarte. Por otro lado, es conveniente aclarar que para predecir el rendimiento escolar en esta población de estudiantes solo bastaría la variable resiliencia, puesto que se considera que la autoestima es una dimensión importante o de las más importantes de la resiliencia.

\section{REFERENCIAS BIBLIOGRÁFICAS}

1. Munist M, Suarez N, Krauskopf D, Silver T. Adolescencia y resiliencia. Buenos Aires: Paidós; 2011.

2. Melillo A, Suárez N. Resiliencia. Descubriendo las propias fortalezas. Buenos Aires: Paidós; 2005.

3. Kotliarenco $M$, Lecannelier F. Resiliencia y subjetividad. Los ciclos de la vida. El apego como mecanismo protector. Buenos Aires: Paidós SAICF; 2006.

4. Saavedra E. Resiliencia: el concepto, un modelo propuesto y su evaluación. Lima: Universidad Nacional de Educación Enrique Guzmán y Valle, Escuela de Postgrado; 2014.

5. Beck U. La sociedad de riesgo. Hacia una nueva modernidad. Buenos Aires: Paidós; 1998.

6. Munist $M$, Suarez $N$. Resiliencia $y$ subjetividad. Los ciclos de la vida. Buenos Aires: Paidós SAICF; 2006.

7. Omar A. Bienestar subjetivo y perspectivas de futuro como predictores de resiliencia en adolescentes. México D.F: Universidad Juárez del Estado de Durango y Centro de investigación y estudios de juventud del Instituto Mexicano de Juventud; 2006.

8. Melillo A. Estamatti $M$, Cuestas A. Resiliencia. Descubriendo las propias 
fortalezas. Buenos Aires: Paidós; 2005.

9. Manciaux M. La resiliencia: resistir y rehacerse. Barcelona: Gedisa; 2003.

10. Muñoz V, De Pedro F. Educar para la resiliencia. Un cambio de mirada en la prevención de situaciones de riesgo social. Revista complutense de educación. 2005; 16(1): 107-124.

11. Anzola M. La resiliencia como factor de protección. Mérida: Universidad de los Andes; 2003.

12. Gallesi R, Matalinares M. Resiliencia y rendimiento académico en estudiantes del 5to y 6to grado de primaria. Revista IIPsi. 2012; 15(1): 181-201.

13. Silas J. La resiliencia en los adolescentes de educación básica, un tema primordial en comunidades marginales. Revista Electrónica Sinéctica. 2008; 31 : 1-32.

14. Henderson N, Milstein M. Resiliencia en la escuela. Buenos Aires: Paidós; 2003.

15. Figueroa D. La resiliencia una alternativa de prevención. Revista de Psiquiatría y Salud Mental. 2005; 6(2): 81-88.

16. Melillo A. Resiliencia. Descubriendo las propias fortalezas. Buenos Aires: Paidós; 2005.

17. Peralta S, Ramírez A, Castrano $H$. Factores resilientes asociados al rendimiento académico en estudiantes pertenecientes a la Universidad de Sucre. Barranquilla: Universidad del Norte; 2006.

18. Villalta $M$. Factores de resiliencia asociados al rendimiento académico en estudiantes de contextos de alta vulnerabilidad. Revista de Pedagogía. 2009; 31 (88): 159-188.

19. Arancibia V. Manual de psicología educacional. Santiago: Universidad Católica de Chile; 1997.

20. Llinares L, Molpeceres A, Musitu G. La autoestima y las prioridades personales de valor. Un análisis de sus interrelaciones en la adolescencia. Anales de psicología. 2001; 17(2): 189-200.

21. Tacconelli G. Nivel de autoestima y el fracaso escolar en alumnos del 8vo grado de la educación básica. [Tesis de licenciatura]. Barquisimeto: Universidad centro occidental "Lisandro Alvarado"; 2006. 